\title{
Fibrillines et fibrillinopathies
}

\section{Gwenaëlle Collod Catherine Boileau}

\section{ADRESSES}

G. Collod : docteur ès sciences. Inserm U. 383, hôpital Necker-Enfants-Malades, Université René-Descartes, Paris V, 149-161, rue de Sèvres, 75743 Paris Cedex 15, France. C. Boileau : maître de conférence, praticien hospitalier. Inserm U. 383, hôpital Necker-Enfants Malades, Université René-Descartes, Paris V, I49-161, rue de Sèvres, 75743 Paris Cedex 15, France, et Laboratoire central de biochimie et de génétique moléculaire, CHU Ambroise Paré, 9, ave nue Charles-de Gaulle, 92104 Boulogne Cedex, France.

TIRÉS À PART

C. Boileau.

Les fibrillines sont les constituants majeurs du réseau microfibrillaire. Cette structure du tissu conjonctif est, soit étroitement associée aux fibres d'élastine, soit présente en agrégats isolés dans les tissus dépourvus d'élastine. Les travaux de biologie cellulaire et moléculaire ont permis d'élucider la structure des fibrillines et de commencer à appréhender leurs relations avec les autres constituants de la matrice extracellulaire. Mais, c'est la description de leur altération dans différentes maladies du tissu conjonctif, dont le syndrome de Marfan, qui a révélé leur importance.

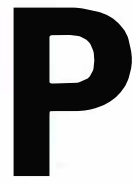
armi les différents constituants de la matrice extracellulaire, les fibres élastiques, comme leur nom l'indique, confèrent au tissu conjonctif ses propriétés élastiques. Ces fibres présentent des assemblages supra-moléculaires constitués de deux éléments: un composant très abondant et amorphe, l'élastine, et un composant microfibrillaire de 10-12 nm localisé à la périphérie de l'élastine, le réseau microfibrillaire (fgure 1). Le terme de microfibrilles a été initialement utilisé pour identifier des agrégats de la matrice extracellulaire morphologiquement similaires et n'ayant pas l'aspect périodique en bandes des fibres interstitielles de collagène [1]. Cette définition grossière a été ensuite restreinte aux microfibrilles de $10 \mathrm{~nm}$ de diamètre qui peuvent s'associer ou non aux fibres élastiques [2]. L'inventaire des constituants macromoléculaires des microfibrilles est actuellement incomplet, en grande partie à cause de leur nature insoluble. Des techniques biochimiques et immunochimiques ont cependant permis, ces dernières années, d'identifier plusieurs macromolécules constitutives des microfibrilles dont les fibrillines.
Les fibrillines sont les constituants majeurs du réseau microfibrillaire. Ce sont de grosses glycoprotéines riches en cystéine, retrouvées tant en association avec l'élastine que dans des structures dépourvues d'élastine comme la zonule*. Deux types de fibrilline sont actuellement identifiés: la fibrilline de type 1 et la fibrilline de type 2. Des mutations dans leurs gènes sont associées non seulement au syndrome de Marfan, prototype des maladies du tissu conjonctif, mais également à toute une série d'affections apparentées, constituant un nouveau groupe de maladies du tissu conjonctif: les fibrillinopathies.

\section{Les fibrillines}

Gène de la fibrilline-I

et structure primaire déduite de la protéine

La fibrilline-1 est codée par un grand gène d'environ $110 \mathrm{~kb}$, le gène $F B N I$ $[3,4]$. L'organisation génomique de FBN1 (limites intron/exon) a été élucidée en 1993 par l'équipe de Pereira [5]. Elle a révélé un gène très morcelé (65 exons) puisque chaque

* Ligament de l'cwil qui maintient en place le cristallin. 


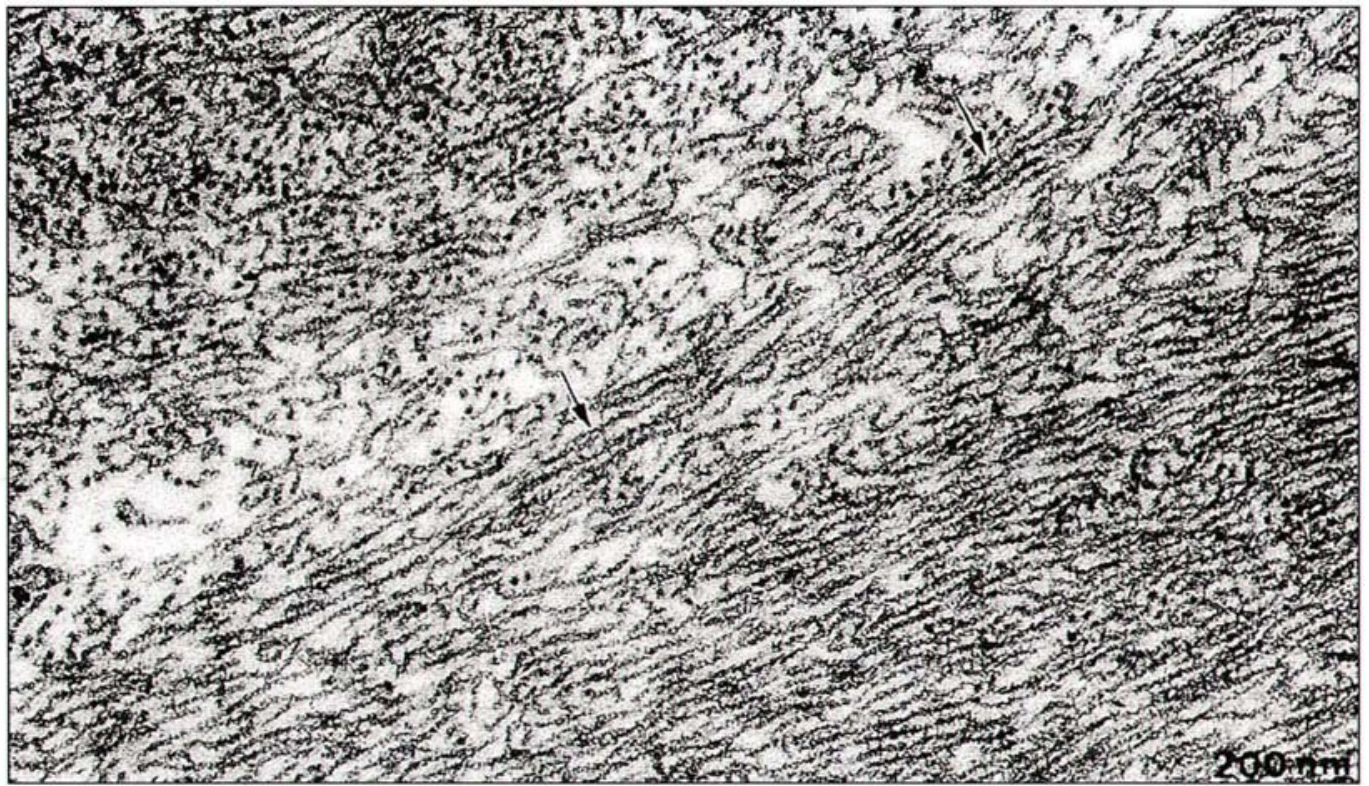

Figure 1. Microscopie électronique après ombrage rotatif représentant le réseau microfibrillaire. Les deux flèches montrent le réseau microfibrillaire en coupe longitudinale (photo aimablement fournie par S. Peyrol, Unité de Pathologie des Fibroses, Institut Pasteur, Lyon).

module est en général codé par un exon. L'ADNc (10 kb environ) a été cloné en deux étapes: tout d'abord sa partie 3' par deux équipes en 1991 $[6,7]$ puis sa partie 5' en 1993 par l'équipe de L. Sakai (Portland, OR, USA) [8]. La structure protéique primaire déduite montre que le gène de la fibrilline code pour une protéine complexe de 2871 acides aminés [5] comprenant de nombreux modules répétés (voir figure 2). La protéine présente des domaines sans homologie avec d'autres protéines: il s'agit des extrémités aminoterminale et carboxyterminale, et de la région riche en proline. Le domaine central de la protéine est formé par la répétition de plusieurs modules. L'analyse comparative a révélé de fortes similitudes entre 47 de ces modules présentant 6 cystéines avec des modules du précurseur de l'EGF (epidermal growth factor) [9]. Ces modules EGF-like sont caractérisés par une organisation conservée de 6 cystéines qui forment des ponts disulfures intrachaînes donnant une conformation en feuillet $\beta$ antiparallèle. Quarante-trois de ces 47 modules contiennent, de plus, la séquence consensus conservée Asp-Asn\AsnGlu\Gln-Asp*`Asn*-Tyr-Phe (où * indique un résidu $\beta$ hydroxylé) qui est nécessaire à la liaison du calcium (module cb EGF-like, cb pour calcium binding). La fibrilline est la première protéine du tissu conjonctif dans été identifiés. En effet, ce module n'avait jusqu'alors été retrouvé que dans des protéines plasmatiques dépendantes de la vitamine $\mathrm{K}$ (facteurs IX et $\mathrm{X}$ de la coagulation, protéines $\mathrm{C}$ et $\mathrm{S}$ ) et dans les protéines Notch, Delta et Crumbs de la drosophile [10]. Un deuxième module comportant 8 cystéines, répété 7 fois dans la protéine, est fortement homologue des modules décrits dans la protéine liant le transforming growth factor- $\beta 1$ (TCF- $\beta$ I BP). Dans la fibrilline-1 comme dans TCF- $\beta 1 \mathrm{BP}$, ces modules à 8 cystéines s'intercalent avec les modules EC(jF-like. Enfin, un troisième module est retrouvé deux fois intercalé dans les répétitions

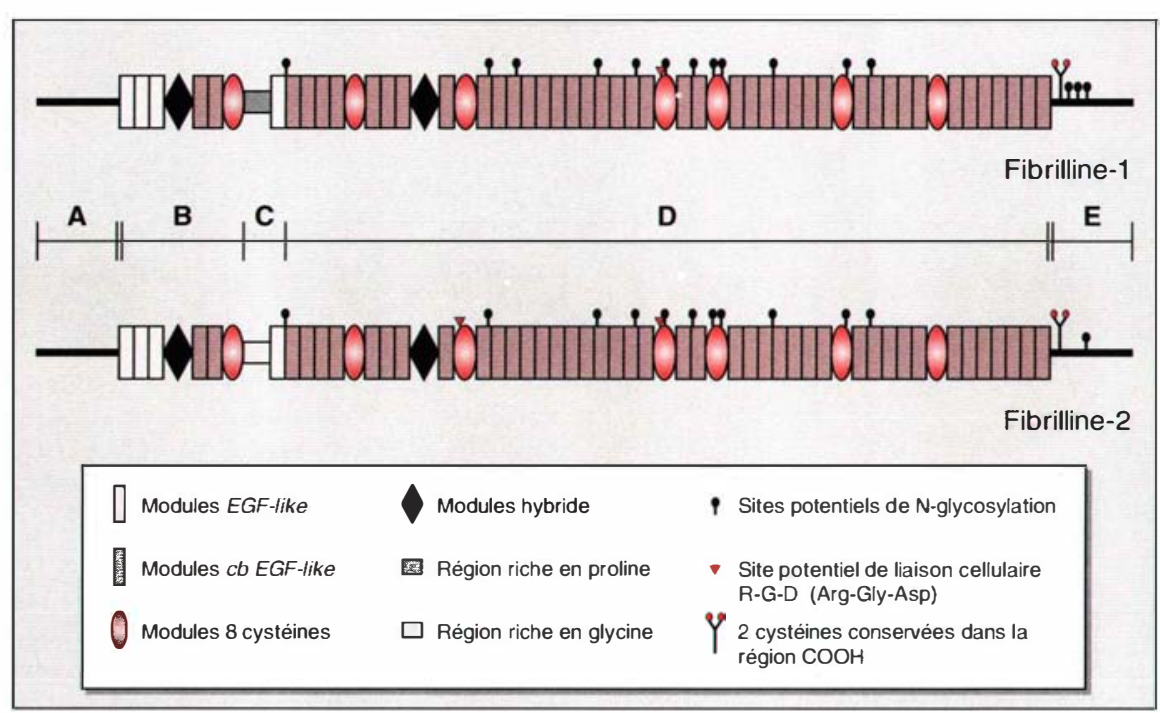

Figure 2. Représentation schématique de la structure protéique primaire déduite de la séquence de l'ADNc de chaque gène de fibrilline. Régions A-B : extrémité aminoterminale. Région $C$ : région riche en proline pour la fibrilline-1 et en glycine pour la fibrilline-2. Région $D$ : domaine central formé par la répétition de plusieurs modules: EGF-like (présentant des similitudes avec le epidermal growth factor), cb EGF-like (EGF-like liant le calcium), "8-cystéines" (présentant des similitudes avec la protéine liant le transforming growth factor- $\beta$ ) et "hybrides" (présentant des similitudes avec les deux premiers modules). Région E: extrémité carboxyterminale. 


\section{RÉFÉRENCES}

1. Low FN. Microfibril: fine filamentous components of the tissue space. Anat Rec $1962 ; 142$ : 131-7.

2. Cleary EG, Gibson MA. Elastin-associated microfibrils and microfibrillar proteins. Int Rev Connect Tiss Res 1983; 10: 97-209.

3. Sakai L, Keene DR, Engvall E. Fibrillin, a new 350-kd glycoprotein, is a component of extracellular microfibrils. J Cell Biol 1986; 103: 2499-509.

4. Sakai LY, Keene DR, Glanville RW, Bächinger HP. Purification and partial characterization of fibrillin, a cysteine-rich structural component of connective tissue microfibrils. $J$ Biol Chem 1991; 266: 14763 70.

5. Pereira L, D'Alessio M, Ramirez F, Lynch, JR, Sykes B, Pangilinan, T Bonadio, J. Genomic organization of the sequence coding for fibrillin, the defective gene product in Marfan Syndrome. Hum Mol Genet 1993; 2: 961-8.

6. Malsen CL, Corson GM, Maddox BK, Glanville RW, Sakai L. Partial sequence of a candidate gene for the Marfan syndrome. Nature 1991; 352 : 3347 .

7. Lee B, Godfrey M, Vitale E, Hori $H$, Mattei MG, Sarfarazi M, Tsipouras $P$, Ramirez F, Hollister D. Linkage of Marfan syndrome and a phenotypically related disorder to two different fibrillin genes. Nature 1991 ; 352 : 330-4.

8. Corson GM, Chalberg SC, Dietz HC Charbonneau NL, Sakai LS. Fibrillin binds calcium and is coded by cDNAs that reveal a multidomain structure and alternatively spliced exons at the 5' end. Genomics 1993; $17: 476-84$.

9. Giry-Lozinguez C, Kleman JP, van der Rest M. Modules et interactions moléculaires au sein des matrices extracellulaires. médecine/sciences $1994 ; 12: 1234-43$.

10. Rao Z, Handford P, Mayhew M, Knott V, Brownlee GG, Stuart D. The structure of a $\mathrm{Ca}^{2+}$-binding epidermal growth factor-like domain : its role in protein-protein interactions. Cell 1995; 82 : 131-41.

11. Raghunath M, Kielty CM, Steinmann B. Truncated profibrillin of Marfan patient is of apparent similar size as fibrillin : intracellular retention leads to over-N-glycosylation. J Mol Biol 1995; 248 : 901-9.

12. Milewicz DM, Grossfield J, Cao SN, Kielty C, Covitz W, Jewett T. A mutation in $F B N 1$ disrupts profibrillin processing and results in isolated skeletal features of the Marfan syndrome. I Clin Invest 1995; 95: 2373-8.
EGF-like et TGF- $\beta 1$ BP. Ces modules, dits "hybrides", présentent des caractéristiques des deux types de modules précédemment décrits (figure 2). Par ailleurs, deux études fonctionnelles [11, 12] ont révélé l'existence d'une pro-fibrilline d'une taille supérieure d'environ $30 \mathrm{kDa}$. Le site de clivage du pro-peptide est encore inconnu mais certainement localisé dans la partie carboxyterminale (région E) [11].

\section{Gène de la fibrilline-2}

et comparaison des structures

primaires déduites des deux gènes

L'existence d'un deuxième gène codant pour une fibrilline a été mise à jour lors des étapes du clonage du gène $F B N 1$. En effet, un clone (MF23) bien que très semblable était localisé sur le chromosome 5 en 5q23 [7]. L'ADNc de ce gène a été caractérisé et, du fait de sa similitude avec le gène $F B N 1$, a été appelé $F B N 2$ et la protéine pour laquelle il code fibrilline-2. La comparaison des deux fibrillines (figure 2) montre une très forte similitude dans la région $\mathrm{D}$ (81\% d'identité), avec une même organisation de 41 modules EGF-like liant le calcium espacés par des modules TGF- $\beta 1$ BP. Tous les sites de $\mathrm{N}$-glycosylation potentiels sont conservés, sauf un. Deux séquences potentielles de liaison cellulaire RGD (Arginine-Glycine-Acide Aspartique) sont retrouvées, dont l'une est identique à la séquence unique localisée dans la fibrilline-1. La région $B$ est également très similaire $(87 \%$ d'identité). La région E (50\% d'identité) diffère par la présence de deux séquences polylysine. Dans la région $\mathrm{A}$ ( $19 \%$ d'identité), une nouvelle séquence riche en proline est présente, juste après le site potentiel de clivage du peptide signal. Malgré cette différence, la région est également basique. La région $\mathrm{C}$ est la plus divergente: très riche en proline dans la fibrilline-1 et en glycine dans la fibrilline- $2[13,14]$.

\section{Assemblage des monomères de fibrilline}

La microscopie électronique après ombrage rotatif de microfibrilles contenant de la fibrilline, isolées de cultures cellulaires à confluence, révèle un aspect en collier de perles foncées, avec une périodicité d'une perle tous les 50-55 nm, séparées par des régions plus claires (figure 3). La base moléculaire de cette périodicité régulière de perles n'est pas connue. Les premières données immunohistochimiques [4] favorisaient l'hypo-

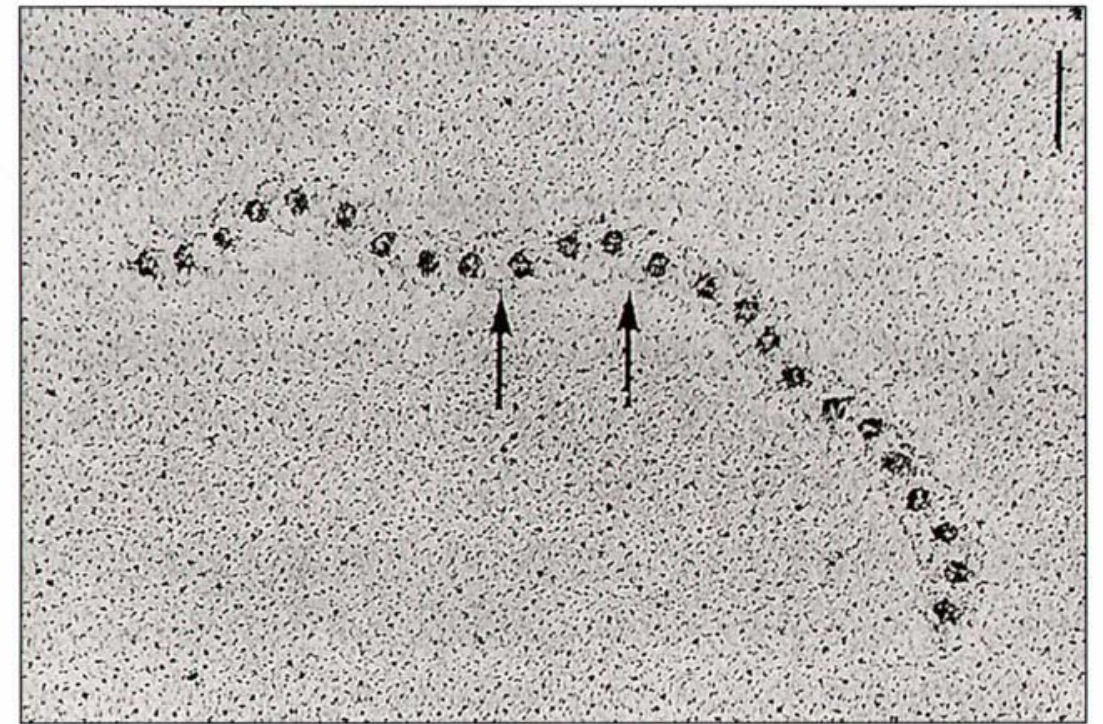

Figure 3. Microscopie électronique après ombrage rotatif de microfibrilles contenant de la fibrilline montrant un aspect en collier de perles foncées, avec une périodicité d'une perle tous les $50-55 \mathrm{~nm}$ (photo aimablement fournie par S. Peyrol, Unité de Pathologie des Fibroses, Institut Pasteur, Lyon). 


\section{RÉFÉRENCES}

13. Zhang $\mathrm{H}$, Apfelroth SD, $\mathrm{Hu}$ W, Davis EC, Sanguineti C, Bonadio J, Mecham RP, Ramirez F. Structure and expression of fibrillin-2, a novel microfibrillar component preferentially located in elastic matrices. $\int$ Cell Biol 1994; 124: 855-63.

14. Zhang H, Hu W, Ramirez F. Developmental expression of fibrillin genes suggests heterogeneity of extracellular microfibrils. $J$ Cell Biol 1995; 129: 1165-76.

15. Kielty CM, Shuttleworth CA. The role of calcium in the organization of fibrillin microfibrils. FEBS Lett 1993; 336 : 323-26.

16. Kielty CM, Berry L, Whittaker SP, Grant ME, Shuttleworth CA. Microfibrillar assemblies of foetal bovine skin. Matrix 1993; 13 : 103-12.

17. Handford P, Downing AK, Rao Z, Hewett DR. The calcium binding properties and molecular organization of epidermal growth factor-like domains in human fibrilfin-1. J Biol Chem 1995; 270: 6751-6.

18. Rosenbloom J, Abrams WR, Mecham RP. Extracellular matrix 4 : the elastic fiber. FASEB J 1993 ; 7 ; 1208-18.

19. Robson P, Wright GM, Sitarz E, Maiti A, Rawat M, Younson JH, Keeley FW. Characterization of lamprin, an unusual matrix protein from lamprey cartilage. $J$ Biol Chem 1993 ; 268 : 1440-7.

20. Collod G, Béroud C, Soussi T, Junien C Boileau C. Software and database for the analysis of mutations in the human $F B N 1$ gene. Nucleic Acids Res 23 : 137-41.

21. McGookey-Milewicz D, Pyeritz RE, Crawford ES, Byers P. Marfan syndrome: defective synthesis, secretion, and extracellular matrix, formation of fibrillin by cultured dermal fibroblasts. $J$ Clin Invest $1992 ; 89$ : 79 . 86.

22. Stahl-Hallengren C, Ukkonen T, Kainulainen $K$, Kristofersson $U$, Saxne T, Tornqvist $\mathrm{K}$, Peltonen $\mathrm{L}$. An extra cysteine in one of the non-calcium-binding-epidermal growth factor-like motifs of the $F B N 1$ polypeptide is connected to a novel variant of Marfan syndrome. I Clin Invest 1994; 94 : 709-13.

23. Grossfield I, Cao S, Milewicz DM. Mutations in the carboxy terminus of FBN1 suggest a potential genotype/phenotype correlation in the Marfan syndrome. Am J Hum Genet 1993; 53: 1167 (abstr)

24. Hewett D, Lynch J, Sykes B. A novel fibrillin mutation in the Marfan syndrome which could disrupt calcium binding of the epidermal growth factor-like module. Hum thèse d'un assemblage en tandem des monomères de fibrilline de façon répétitive le long de la microfibrille mais l'organisation exacte ainsi que les interactions moléculaires restaient indéterminées. La potentialité de la fibrilline à lier le calcium, fortement suggérée par la présence en abondance des modules EGF-like possédant des séquences consensus de liaison du calcium (cb E(FF-like), a récemment été démontrée [8]. L'identification chez des patients attein ts d'un syndrome de Marfan de mutations dans des parties du gène de la fibrilline codant pour ce type de domaine, prédisant une anomalie de liaison du calcium, souligne l'importance potentielle de la liaison du calcium pour l'intégrité de la microfibrille et sa fonction. Des expériences d'ajout ou de chélation de calcium $[15,16]$ ont montré un impact majeur sur l'organisation des régions entre les perles alors que, de façon surprenante, l'intégrité des perles, leur périodicité ainsi que la longueur des microfibrilles ne semblent pas affectées. Ces observations indiquent que la région riche en modules $c b$ EGF-like constitue l'élément structural majeur des régions interperles. Le rôle du calcium serait d'induire un changement structural local de regroupement latéral et d'alignement des monomères de fibrilline adjacents au niveau des domaines interperles. Le fait que le calcium soit en position relativement interne dans la structure, suggère que sa liaison au module EGF-like aurait plutôt un rôle stabilisateur au niveau de chaque monomère de fibrilline plutôt que d'intervention dans des liaisons reliant des monomères de fibrilline entre eux [17].

Des expériences récentes de cristallographie en phase liquide de modules cb EGF-like appartenant à différentes protéines $[10,17]$ ont montré que la succession de modules EGF-like au sein de chaque monomère engendrait un arrangement hélicoïdal qui serait stabilisé par le calcium. En se fondant sur ce modèle, Handford $e$ t al. en 1995 [17] ont proposé un modèle d'interaction entre plusieurs monomères de fibrilline: une première étape serait la dimérisation de deux molécules de fibrilline dans une orientation antiparallèle, suivie de l'association des deux dimères, pour former un corps de quatre hélices (figure 4). L'étape à franchir maintenant est la validation de ce modèle ainsi que l'élucidation des interactions de la fibrilline avec les autres composants du réseau microfibrillaire. Ces interactions pourraient se situer au niveau des perles et expliquer leur stabilité vis-à-vis des variations (ajout et chélation) de calcium.

Interaction de la fibrilline avec les autres constituants du réseau microfibrillaire, la matrice extracellulaire, et l'élastine

L'inventaire des constituants des microfibrilles est actuellement incomplet. Plusieurs macromolécules constitutives des microfibrilles, autres que les fibrillines, ont cependant pu être identifiées: les quatre MFAP (microfibril-associated protein), la lysyl oxydase, l'émiline ou gpl15 (glycoprotéine $115 \mathrm{kDa}$ ), et la gp36 (glycoprotéine $36 \mathrm{kDa}$ ). Les fonctions des différentes protéines au sein de ce réseau ainsi que les relations entre ses différents constituants ne sont pas encore élucidées et les interrelations entre les fibrillines et les autres protéines composant la matrice extracellulaire restent obscures. Le réseau microfibrillaire semble, cependant, jouer un rôle crucial dans l'élastogenèse, servant d'échafaudage sur lequel se déposerait l'élastine. L'hypothèse classique est que les molécules de tropoélastine sont sécrétées puis diffusent simplement à la surface de fibres en croissance sur lesquelles elles se lieraient tout en étant reliées

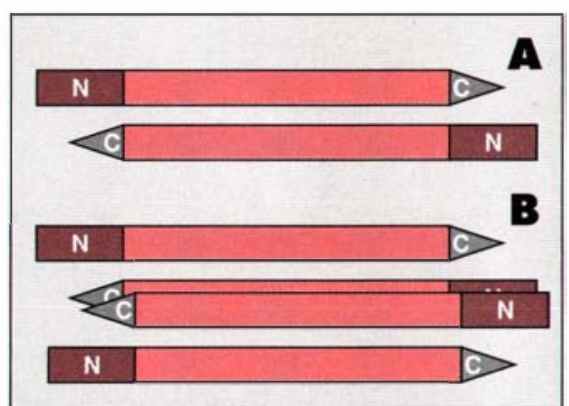

Figure 4. Modèle de polymérisation des monomères de fibrilline. A. dans une première étape dimérisation par association tête à queue. B. puis association de deux dimères pour former un corps à quatre hélices (D'après Handford et al. [17].) 


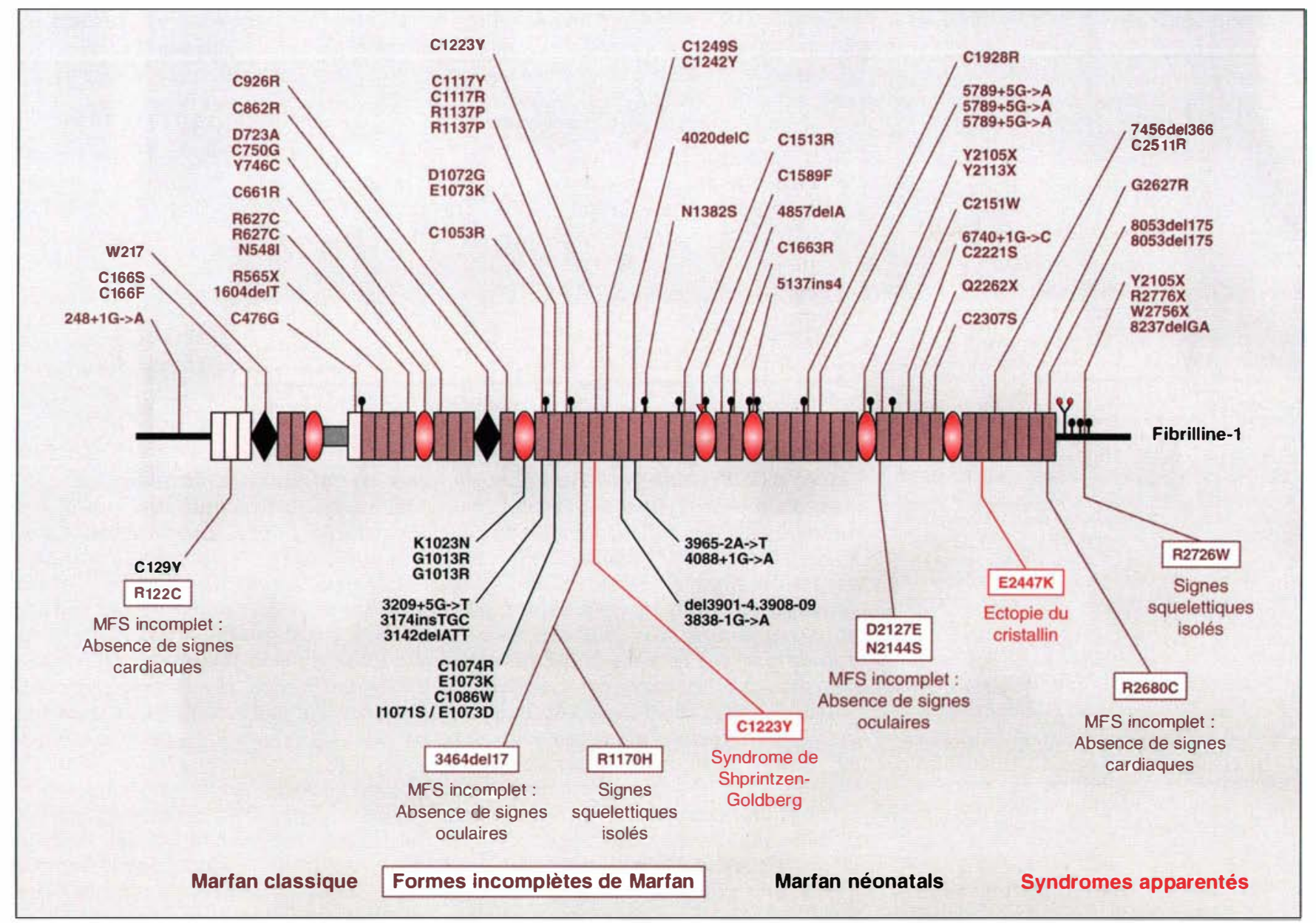

Figure 5. Représentation schématique des 76 mutations du gène FBN1 rapportées en fonction de leur répartition dans les différents types de modules de la protéine. Certaines mutations ont été observées plusieurs fois chez des individus différents ou associées à des pathologies différentes.

Code à une lettre des acides aminés: A: Ala;C:Cys;D:Asp; E: Glu; F: Phe; G: Gly; H: His; I: Ile; K: Lys; L: Leu; M: Met; N: Asn; P: Pro; R: Arg; S: Ser; T:Thr; V: Val; W: Trp; Y: Tyr.

entre elles; mais ce modèle ne permet pas d'expliquer l'efficacité du processus d'assemblage ni la variété de formes des fibres élastiques dans différents tissus. Il semble évident aujourd'hui que la sécrétion de tropoélastine ainsi que son assemblage nécessitent l'aide d'autres protéines aussi bien à l'intérieur de la cellule qu'à l'extérieur. Les fibrillines constituent de très bons candidats. La fibrillogenèse de l'élastine s'effectue en effet près de la membrane. Les microfibrilles sont le premier composant visible des fibres élastiques, apparaissant sous la forme de petits amas près de la membrane plasmique. Lors du développement des fibres élastiques, l'élastine apparaît comme un matériel amorphe, au sein des amas microfibrillaires. Progressivement, ces régions amorphes s'assemblent pour former le coeur central d'élastine. Les microfibrilles sont alors majoritairement et progressivement déplacées tout autour de la fibre, position qu'elles conservent dans les tissus à maturité. Le fait que les agrégats de microfibrilles prennent la forme et l'orientation des futures fibres élastiques suggère que ce réseau permettrait d'aligner les molécules de tropoélastine de façon à juxtaposer les sites de liaison entre ces molécules avant oxydation par la lysyl oxydase [18].

\section{Expression des gènes de fibrillines au cours de l'embryogenèse et localisation chez l'adulte}

L'expression des gènes de fibrillines durant le développement a été étu- diée chez la souris et montre un profil caractéristique en deux temps, avec une transcription de $F B N 2$ apparaissant plus précocement que celle de FBN1. Hormis le système cardiovasculaire où l'activité du gène $F B N 1$ est plus précoce et toujours plus élevée que celle de $F B N 2$, il semble exister dans les autres systèmes une corrélation entre le moment de l'expression de chaque gène de fibrilline et les différents stades de la morphogenèse. En effet, l'accumulation des transcrits de $F B N 2$ atteint un plateau juste avant la différenciation tissulaire effective et décroît rapidement après, voire même disparaît. Au contraire, la quantité de transcrits $F B N 1$ s'accroît de façon graduelle tout au long de la morphogenèse [14]. 


\section{RÉFÉRENCES}

25. Kainulainen K, Karttunen L, Puhakka L, Sakai L, Peltonen L. Mutations in the fibrillin gene responsible for dominant ectopia lentis and neonatal Marfan syndrome. Nature Genet 1994; 6: 649.

26. Tynan K, Comeau K, Pearson $\mathbf{M}$, Wilgenbus $P$, Levitt D, Gasner C, Berg $M$, Miller D, Francke U. Mutation screening of complete fibrillin-1 coding sequence: report of five new mutations, including two in 8-cysteine domains. Hum Mol Genet 1993; $2: 1813-21$

27. Milewicz DM, Grossfield J, Cao SN, Kielty C, Covitz W, Jewett T. A mutation in $F B N 1$ disrupts profibrillin processing and results in isolated skeletal features of the Marfan syndrome. I Clin Invest 1995; 95 : 2373-8.

28. Hayward C, Porteous MEM, Brock DJH. A novel mutation in the fibrillin gene (FBN1) in familial arachnodactyly. Mol Cell Probe 1994; 8: 325-7.

29. Boileau C, Jondeau G, Babron MC, Coulon M, Alexandre JA, Sakai L, Melki J, Delorme G, Dubourg $O$, Bonaïti-Pellié $C$, Bourdarias JP, Junien C. Autosomal dominant Marfan-like connective-tissue disorder with aortic dilation and skeletal anomalies not linked to the fibrillin genes. Am J Hum Genet 1993; 53 : 46-54

30. Collod G, Babron MC, Jondeau G, Coulon M, Weissenbach J, Dubourg $O$ Bourdarias IP, Bonaīti-Pellié C, Junien C, Boileau C. A second locus for Marfan syndrome maps to chromosome 3p24.2-p25. Nature Genet 1994 ; 8: 264-8.

31. Milewicz DM, Cho M, Zinn AB, Towbin JA, Byers PH, Putnam EA. Delineation of the Marfan phenotype associated with mutations in exons 23 . Am J Hum Genet 1995; 57 : A53, abstract 277.

32. Lonngvist $\mathrm{L}$, Child A, Kainulainen $\mathrm{K}$, Davidson R, Puhakka L, Peltonen L. A novel mutation of the fibrillin gene causing ectopia lentis. Genomics $1994 ; 19: 573-6$.

33. Francke U, Berg MA, Tynan K, Brenn T, Liu W, Aoyama T, Gasner C, Miller DG, Furthmayr H. A Gly1127Ser mutation in an EGF-like domain of the fibrillin-1 gene is a risk factor for ascending aortic aneurysm and dissection. Am J Hum Genet 1995; 56 :

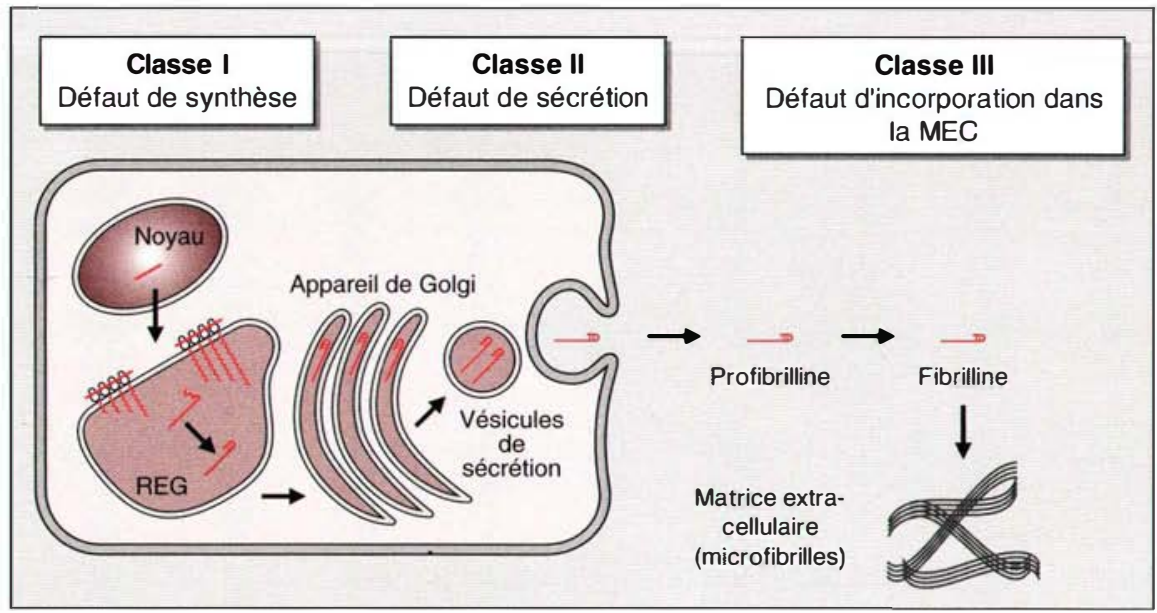

Figure 6. Différentes classes fonctionnelles de défaut de la fibrilline-1. L'anaIyse du métabolisme de la fibrilline-1 chez différents sujets atteints d'une forme classique du syndrome de Marfan révèle 4 classes fonctionnelles différentes de défaut. Classe I: altération quantitative de la synthèse: la quantité de fibrilline produite est de $50 \%$ par rapport aux cellules témoins. L'allèle sauvage est donc normalement synthétisé tandis que l'allèle muté, soit ne donne aucun produit, soit donne un produit tronqué qui n'est pas sécrété ou qui est présent en très faible quantité (diminution de la stabilité du transcrit, sécrétion inefficace ou dégradation intracellulaire du produit muté). Classe II : altération de la sécrétion de fibrilline. Dans ces cellules, la quantité de fibrilline produite est normale mais sa sécrétion dans le milieu de culture est très retardée par rapport aux contrôles et son incorporation dans la matrice extracellulaire est médiocre. Dans ce groupe fonctionnel, les molécules mutantes semblent bloquer par un mécanisme "dominant-négatif" la polymérisation car la quantité de matériel déposé est plus faible que dans la classe I. Classe III: la synthèse et la sécrétion de fibrilline sont normales mais il y a une forte diminution d'incorporation dans la matrice extracellulaire (MEC). Les mécanismes moléculaires sous-jacents sont probablement hétérogènes. Enfin, dans le dernier groupe (IV) aucune anomalie de synthèse, de sécrétion ou d'incorporation à la matrice extracellulaire n'a pu être mise en évidence, argument en faveur de l'hétérogénéité génétique du syndrome de Marfan (démontrée par une approche génétique [30]). REG: réticulum endoplasmique rugueux.

Le gène $F B N 2$ est exprimé principalement durant l'embryogenèse dans une grande variété de tissus et de cellules, comprenant le mésenchyme, l'épithélium, les chondrocytes ainsi que les cellules vasculaires, squelettiques et myocardiques. Ce profil mésodermique d'expression contraste avec le fait que ce gène n'a été découvert que durant l'étape de clonage du gène $F B N 1$. Il semble maintenant fort probable que l'âge des tissus utilisés alors ait été la principale cause de l'ignorance de l'existence d'une deuxième fibrilline [14].

Chez l'adulte, l'utilisation d'anticorps antifibrilline permet de révéler une distribution étendue des fibrillines dans la matrice extracellulaire, soit associées à l'élastine (peau, poumons, reins, vaisseaux, cartilages, tendons, muscles), soit dans des tissus ne contenant aucune fibre d'élastine, comme la zonule ciliaire. L'identification d'une région protéique très divergente entre les deux fibrillines, la région $\mathrm{C}$ (figure 2), a permis de fabriquer des anticorps spécifiques de chacune des protéines. Leur utilisation révèle une distribution très semblable des deux fibrillines avec une localisation préférentielle de la fibrilline-2 pour les matrices extracellulaires riches en fibres élastiques [14]. Certaines différences on t cependant pu être notées. Au niveau de la peau, la fibrilline-l forme des filaments discrets reliant le derme à 
la jonction épidermique tandis que la fibrilline-2, bien qu'ayant la même localisation, est plus abondante dans le derme, particulièrement autour des follicules pileux et des glandes sudoripares [13].

\section{Fonction des fibrillines dans le réseau microfibrillaire}

Étant donné la diversité des profils spatio-temporels de l'expression des deux gènes de fibrilline, il est fort possible que les deux protéines jouent des rôles différents. La protéine fibrilline-2 est préférentiellement trouvée dans les tissus riches en élastine comme le cartilage, la média de l'aorte et le long de l'arbre bronchique. Durant l'embryogenèse, la synthèse de la fibrilline-2 est plus précoce que celle de la fibrilline-1 et est apparemment majoritaire pendant la courte période de temps précédant l'élastogenèse. Il est donc possible qu'une des fonctions majeures de la fibrilline-2 durant la morphogenèse précoce soit de participer à l'orientation de l'élastine lors de l'assemblage des fibres élastiques. En effet, la région riche en glycine de la fibrilline-2, très hydrophobe, montre des similitudes avec de nombreux fragments de l'élastine [18]. Comme eux, cette région peut donc théoriquement former des feuillets $\beta$ et des coudes $\beta$ facilitant l'agrégation protéique par des interdigitations des parties hydrophobes des chaînes [19]. Il est donc possible que cette région de la fibrilline- 2 relaie des interactions décisives avec l'élastine durant l'assemblage précoce des fibres élastiques.

Inversement, l'expression plus tardive de $F B N 1$ a u cours du développement et la prédominance de la fibrilline-1 dans les structures responsables de la résistance à la charge et à la tension (comme l'adventice de l'aorte, les ligaments suspenseurs du cristallin, la peau) suggèrent que cette glycoprotéine est principalement responsable de la fonction structurale des microfibrilles.

\section{Les fibrillinopathies}

Très rapidement après le clonage des gènes $F B N 1$ et $F B N 2$, de nombreux travaux ont montré que des altérations de ces gènes étaient associées à différentes maladies du tissu conjonctif. Ces maladies, longtemps considérées comme des collagénopathies, constituent le nouveau groupe des fibrillinopathies.

\section{Fibrillinopathies de type 1 ou maladies de la fibrilline du chromosome 15 (FBN1)}

A ce jour 76 mutations du gène $F B N 1$ ont été décrites (voir pour revue [20] et figure 5). Leur analyse révèle une absence totale de gros réarrangement dans le gène. Ces mutations sont réparties sur toute la longueur du gène et sont dans leur grande majorité "privées" (seulement 7 cas de mutations récurrentes sont décrits). Il s'agit surtout de mutations faux-sens $(50 / 76$ soit $65 \%$ des mutations) touchant préférentiellement des cystéines (26/50 soit $52 \%$ des mutations). Seules 6 mutations nonsens (8\% des mutations) ont été décrites. Les décalages du cadre de lecture ne sont associés qu'à $27 \%$ des mutations $(21 / 76)$ avec une égalité de répartition entre les mutations de sites d'épissage $(9 / 76)$ et les délétions $(10 / 76)$, les mutations de type insertion étant minoritaires $(2 / 76)$. Dans $72,7 \%$ des cas $(56 / 76)$, elles sont localisées dans un module $c b$ EGF-like. Cela n'est pas surprenant puisque ces modules représentent plus des trois quarts de la protéine. Les autres mutations sont retrouvées dans tous les autres domaines sauf le domaine riche en proline (dans lequel aucune mutation n'a été identifiée à ce jour). Les mutations du gène FBN1 sont associées tant au syndrome de Marfan, prototype des maladies du tissu conjonctif, qu'à un ensemble d'affections présentant, soit de façon isolée certains symptômes du syndrome de Marfan (ectopie du cristallin, signes squelettiques ou forme familiale d'anévrysme), soit des symptômes n'appartenant pas au spectre de ce syndrome (formes néonatales de syndrome de Marfan, syndrome de Shprintzen-Golberg, syndrome de Weill-Marchesani).

Marfan classique (OMIM \#154700) et formes incomplètes du Marfan

Le syndrome de Marfan est une maladie autosomique dominante qui atteint dans sa forme classique trois systèmes: (1) squelettique (défor- 
mations du thorax, dolichosténo-

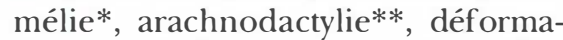
tions rachidiennes, grande taille, déformations de la voûte palatine, vice architectural de hanche et mobilité articulaire anormale); (2) oculaire (dislocation du cristallin, décollement de la rétine, myopie) et (3) cardiovasculaire (dilatation aortique, anévrysme, dissection aortique, prolapsus valvulaire mitral). Il existe des formes incomplètes où l'atteinte oculaire ou l'atteinte cardiovasculaire n'est pas retrouvée. L'expressivité intrafamiliale ou interfamiliale est en outre très variable. On estime actuellement que le syndrome de Marfan touche une person ne sur 10000 individus. Cependant, la grande variabilité phénotypique, l'absence d'un test diagnostic sensible et spécifique, génétique ou biochimique, ainsi qu'un fort taux de cas sporadiques suggèrent que l'incidence du syndrome de Marfan est en réalité beaucoup plus élevée. Il représente la deuxième maladie la plus fréquente du tissu conjonctif après l'ostéogenèse imparfaite.

A ce jour, 52 mutations dans le gène FBN1 responsables de syndrome de Marfan classique ont été décrites. Elles sont majoritairement localisées dans des domaines EGF-like liant le calcium (38/52 soit $73 \%$ des cas), les autres types de domaines étant peu atteints (cinq dans des modules à 8 cystéines, deux dans des modules EGF-like, deux dans des modules "hybride", un dans la région aminoterminale unique, et quatre dans la région carboxyterminale unique). Des tests effectués sur des fibroblastes de certains patients ont révélé quatre classes fonctionnelles de défauts (figure 6) [21]. Néanmoins très peu d'auteurs ont recherché simultanément le défaut moléculaire et son effet sur le métabolisme de la fibrilline-1. Il est donc impossible actuellement de prévoir la classe de défaut fonctionnel à laquelle rattacher une mutation donnée.

Plusieurs mutations ont été rapportées pour des patients présentant des formes incomplètes de syndrome de Marfan: (1) Marfan sans signes car-

* Longueur el minceur anormales des membres.

** Longueur el minceur anormales des doigts el des diaques pour R122C*** (EGF-like \#2****) [22] et R2680C ( cb liGF-like \#43) [23] alors que des mutations dans ces mêmes domaines provoquent dans les autres cas une atteinte cardiaque. (2) Marfan sans signes oculaires pour N2144S ( $c b$ EGF-like \#32) [24], D2127E (cb EGF-like \#32) [25], et 3464del17 (cb EGF-like \#14) [26]. (3) Enfin les mutations R2726W (région carboxyterminale unique) [27] et R1170H (cb EGF-like \# 14) [28] ont été identifiées chez des patients présentant de façon isolée les signes squelettiques caractéristiques du syndrome de Marfan.

Enfin, il est à noter que les défauts du gène $F B N 1$ ne rendent pas compte de toutes les formes de syndrome de Marfan. En effet, par l'étude d'une grande famille française présentant une forme incomplète de ce syndrome, nous avons démontré l'existence d'une hétérogénéité génétique. Dans cette famille, la maladie n'est pas liée à une mutation du gène FBN1 [29] mais à une mutation au locus MFS2 en 3p24.2-p25 [30]. Le gène $M F S 2$ est actuellement inconnu : il ne correspond à aucune des fibrillines déjà identifiées, ni à l'un des composants du réseau microfibrillaire actuellement connus. Il est très probable que $M F S 2$ code, soit pour un constituant du réseau microfibrilllaire, soit pour une protéine interagissant avec celui-ci. L'identification de MFS2 est actuellement en cours par une approche de clonage positionnel.

\section{Marfan néonatal ou infantile}

Il s'agit d'une forme plus précoce et plus sévère de syndrome de Marfan impliquant des pertes de mobilité des articulations comme celles retrouvées dans l'arachnodactylie contracturante congénitale (CCA) (ou syndrome de Beals-Hecht, lié à $F B N 2$ ), un faciès anormal, des maladies plurivalvulaires et une mort précoce généralement due à une insuffisance cardiaque et non à un anévrysme aortique, cause la plus fréquente de décès dans le syndrome de Marfan. L'association de signes du

\footnotetext{
*** Code à une leltre des acides aminés, voir légende de la figure 5 .

***** La localisation de chaque mulation dans un des modules conservés est indiquée par le numéro de ce dernier.
}

Marfan et du CCA a longtemps fait supposer qu'il ne s'agissait pas d'une anomalie du gène FBN1. En 1994, l'équipe de L. Peltonen a donné, par la description de plusieurs mutations, la preuve de l'implication de ce gène dans cette forme particulière de syndrome de Marfan [25]. Depuis, 15 mutations ont été rapportées (figure 5), localisées dans 14 cas sur 15 dans les exons 24 à 32 de la protéine (11 mutations dans les modules $c b$ liGF-like\#11 à 18 et 3 dans le module à 8 cystéines \#3). Cette région est liée également à des formes sévères de syndrome de Marfan caractérisées par des faciès anormaux et une absence de perte de mobilité des articulations [31].

\section{Ectopies du cristallin (OMIM 129600)}

Des cas familiaux de dislocation du cristallin avec une transmission autosomique dominante ont été rapportés, avec ou sans anomalies squelettiques modérées, mais sans anomalies cardiovasculaires. Une seule mutation, E2447K, a été décrite dans une de ces familles impliquant le module cb EGF-like \#38 [25, 32]. Il s'agit de la seule mutation décrite à ce jour dans ce domaine.

\section{Formes familiales d'anévrysme}

Une seule variation (G1127S) dans le motif $c b$ EGF-like \#13 a été rapportée jusqu'à présent [33]. Elle a été décrite dans une famille présentant une transmission autosomique dominante d'anévrysmes sans aucun autre symptôme du syndrome de Marfan. Néanmoins, la nature délétère de cette variation n'a pas été formellement démontrée puisque l'un des sujets atteints ne la possédait pas. Cela suggérerait qu'il s'agirait plutôt d'un facteur de risque. Cette hypothèse est étayée par les données de l'équipe de L. Peltonen (Helsinki, Finlande) qui a exclu par analyse familiale l'implication du gène $F B N 1$ dans différentes familles scandinaves présentant des anévrysmes (L. Peltonen, communication personnelle). Enfin, très récemment, l'implication du gène $F B N 1$ a été démontrée dans deux maladies très rares: le syn- 
drome de Shprintzen-Goldberg et le syndrome de Weill-Marchesani.

\section{Syndrome de Shprintzen-Goldberg (OMIM 182212)}

Ce syndrome peut associer différentes manifestations du syndrome de Marfan associées à une craniosynostose ou à d'autres anomalies crânio-faciales telles que l'hypoplasie malaire ou les orbites peu profondes, une laxité de l'oropharynx, une hypotonie infantile, un retard de développement et un retard mental. Tous les cas rapportés sont sporadiques. Une mutation a été décrite chez un patient de 7 ans dans le module cb EGF-like \#15, C1223Y [34]. De façon surprenante, cette même mutation avait déjà été rapportée dans un cas de syndrome de Marfan classique [35]. Cela laisserait supposer que ce syndrome représenterait un cas de digénisme dont la mutation dans le gène $F B N 1$ ne serait que l'une des composantes.

\section{Syndrome de Weill-Marchesani (OMIM 227600)}

Ce syndrome comprend des anomalies squelettiques telles que petite taille, lordose, perte de mobilité au niveau des mains, anomalies oculaires telles que ectopie du cristallin et myopie. Dans une famille comprenant trois générations et présentant une transmission autosomique dominante de ce syndrome, des anomalies de la fibrilline ont été mises en évidence in vitro sur des cultures de fibroblastes. La mutation exacte du gène $F B N 1$ n'a pas encore été identifiée [36].

Actuellement, aucune corrélation entre la localisation d'une mutation dans le gène FBN1 et un (ou des) signe (s) clinique(s) particulier(s) n'a pu être mise en évidence. I.a seule exception concerne les mutations situées dans les exons 24 à 32 qui semblent impliquées non seulement dans les formes néonatales de syndrome de Marfan mais également dans des formes sévères particulières caractérisées par des faciès anormaux et une absence de contracture. La diversité et la gravité variable des signes cliniques associés aux mutations du gène $F B N 1$ restent mal expliquées. Elles sont certainement le reflet non seulement de la répartition ubiquitaire de la fibrilline-1, mais aussi d'une hétérogénéité des structures microfibrillaires vraisemblablement liée à une spécificité tissulaire et fonctionnelle.

\section{Fibrillinopathies de type 2 ou maladies de la fibrilline du chromosome 5 (FBN2)}

A l'inverse de la diversité des maladies liées à la fibrilline de type 1 , les altérations du gène $F B N 2$ ne sont actuellement associées qu'à deux maladies très rares:

L'arachnodactylie contracturante congénitale (CCA) ou syndrome de Beals-Hecht (OMIM 121050) : Ce syndrome comprend les signes squelettiques du syndrome de Marfan tels que l'arachnodactylie, la dolichosténomélie et les déformations rachidiennes comme la scoliose, associés à des contractures congénitales et des anomalies de l'oreille externe. Aucun des signes oculaires et cardio-vasculaires caractéristiques du syndrome de Marfan n'est présent. Jusqu'à ce jour, seules deux mutations du gène $F B N 2$ ont été décrites E390K [37] et C1252Y [38]. Anecdotiquement, il est intéressant de noter que la malade initialement décrite par Marfan en 1896 aurait plutôt été atteinte de CCA que d'un véritable syndrome de Marfan [39].

\section{Un nouveau syndrome}

à transmission autosomique dominante

L'équipe de D. Milewicz a rapporté dans une famille de 15 membres (dont 8 sont atteints) la ségrégation d'un nouveau phénotype de transmission autosomique dominante comprenant arachnodactylie, grande taille, scoliose, hypertélorisme*, front saillant, profil facial plat, pertes momentanées de flexion des extrémités des doigts mais hypermobilité des articulations telles que poignets, hanches ou épaules, et perte d'audition. Une analyse familiale avec des marqueurs intragéniques a permis d'impliquer le gène FBN2 dans cette nouvelle maladie [40]. Il semble donc que les mutations du gène FBN2 soient responsables d'un spectre clinique plus étroit associé à

* Élargissement anormal de l'espace inter culaire. un tropisme squelettique et articulaire, sans atteinte cardiaque ni oculaire.

\section{Conclusion}

Les travaux de biologie cellulaire puis moléculaire de ces dernières années ont permis de commencer à dresser l'inventaire des composants du réseau microfibrillaire. Parallèlement, l'implication des altérations des fibrillines dans différentes maladies humaines a révélé le rôle structural majeur de ces protéines et a ouvert un nouveau champ d'investigation de leurs fonctions grâce à la disponibilité de nombreux mutants naturels. Parmi ceux-ci, les mutants des domaines cb EGF-like devraient permettre de mieux comprendre le rôle, tant structural que fonctionnel, de ce type de module dans une protéine du tissu conjonctif. Le défi à relever maintenant est double: achever l'inventaire des composants du réseau microfibrillaire et comprendre comment ils s'assemblent et interagissent. La diversité des altérations tissulaires observées dans les fibrillinopathies laisse déjà supposer l'existence d'une hétérogénéité structurale du réseau, associée vraisemblablement à une spécificité fonctionnelle. Il est donc fortement probable que dans les années à venir les fibrillinopathies ne représenteront qu'un groupe d'affections dans un ensemble plus large de "maladies du réseau microfibrillaire»

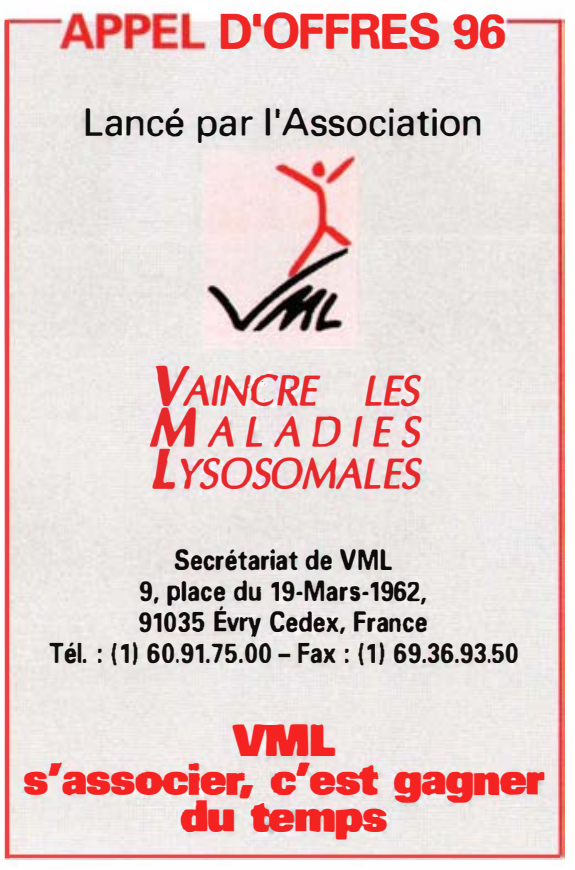




\section{Summary}

Fibrillins and fibrillinopathies

Microfibrils contain a variety of proteins, the most prominent of which are the two fibrillins. Fibrillins are large glycoproteins (320 kDa) ubiquitously distributed in connective tissues. Together with amorphous elastin, fibrillin-containing microfibrils form the elastic fibers. Fibrillins are also found in microfibrils that are apparently unendowed with amorphous elastin, such as those forming the ciliary zonule. Fibrillins are encoded by two different genes: FBN 1 on human chromosome $15 q 21$ encodes type 1 fibrillin and FBN2 on human chromosome $5 \mathrm{q} 23$ encodes type 2 fibrillin. The most notable structural feature of these proteins is the presence of numerous calcium-binding epidermal growth-factor-like motifs interspersed with a few motifs homologous to those found in the binding protein for transforming growth factor $\beta$. The study of the developmental expression of the fibrillin genes has revealed different patterns: $F B N 1$ is mainly expressed during late morphogenesis while $F B N 2$ expression coincides with early morphogenesis and notably the beginning of elastogenesis. Therefore, fibrillins could contribute to the structural and functional heterogeneity of microfibrils. This heterogeneity is reflected in the large phenotypic spectrum associated with mutations in the fibrillin genes. These mutations are found in a new group of inherited connective-tissue disorders: the fibrillinopathies. To date, 76 mutations have been identified in the FBN1 gene. These mutations are associated not only with the Marfan syndrome (MFS) but also with a spectrum of conditions phenotypically related to MFS including dominantly inherited ectopia lentis, severe neonatal Marfan syndrome and isolated typical features of Marfan syndrome. Conversely, only a few mutations have been reported in the FBN2 gene and they are observed in congenital contractural arachnodactyly. Important issues that will now have to be addressed concern the elucidation of the exact composition of the microfibrils and their spatial organization into tissuespecific macro-aggregates.

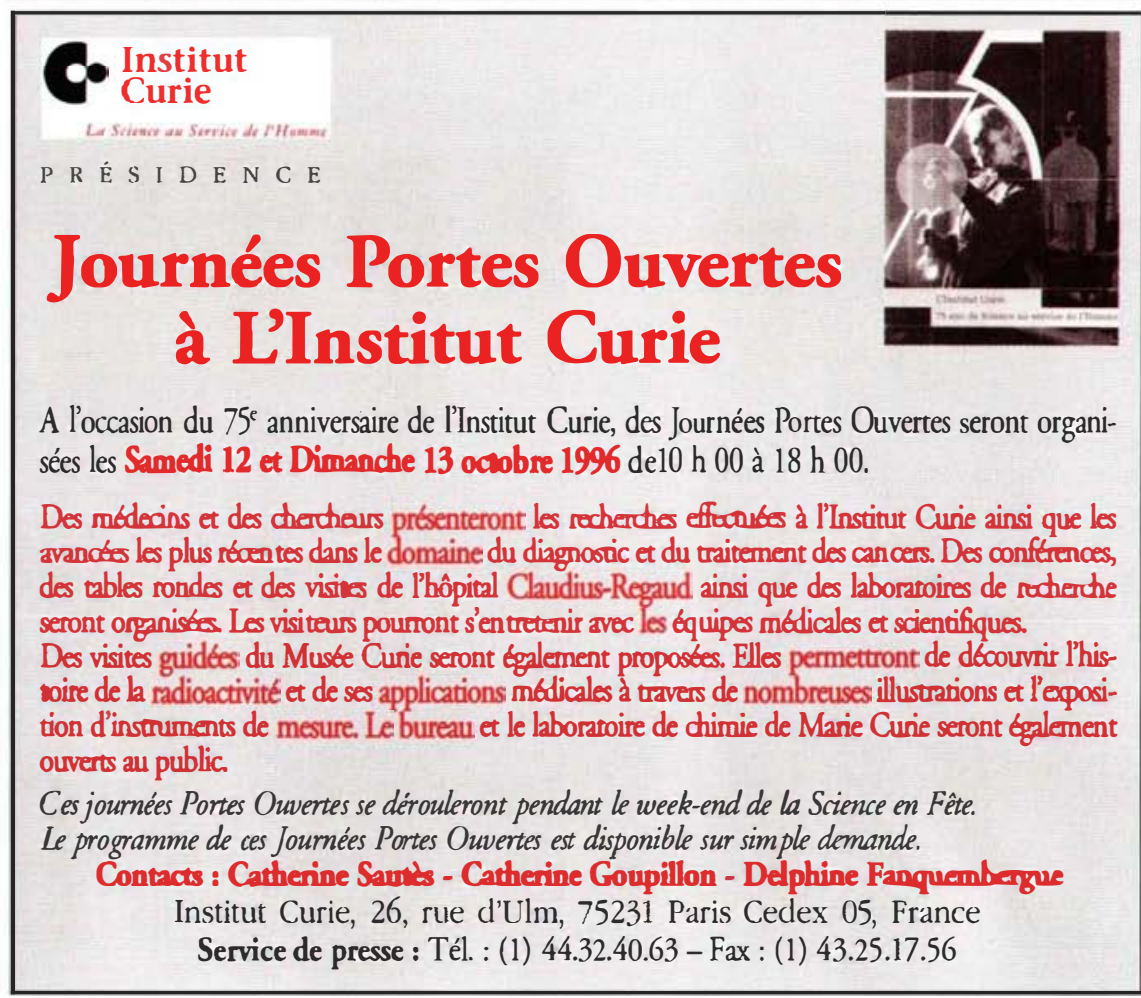

\section{Le nouveau Rosenwald 1996}

\section{Tout savoir sur les médecins, les établissements de soins et les professionnels de la santé}

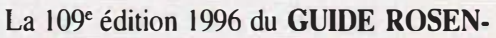
WALD vient de paraître en 3 volumes de 6004 pages soit 1244 pages de plus qu'il y a 3 ans ! C'est un véritable record!

Cette année encore, le Rosenwald 1996 contient de nombreuses améliorations de contenu et de forme pour mieux satisfaire les besoins du corps médical français.

- Le Tome 1 (2 264 pages) fournit tous les renseignements sur les 162808 médecins hospitaliers et libéraux, classés par ordre alphabétique pour la France entière. - Le Tome 2 (2 200 pages) reclasse les médecins par ordre géographique et par spécialités.

- Le tome 3 (1 540 pages) regroupe les généralistes classés par département et les partenaires de la Santé : administrations, laboratoires et fournisseurs, hôpitaux et cliniques.

Plus de la moitié des fiches biographiques et professionnelles des 162808 médecins et des 11271 établissements de soins recensés sont corrigées par les médecins et établissements eux-mêmes d'une édition à l'autre. Ces infornations fiables, utiles et indispensables, en permanence à portée de votre main, introuvables ailleurs, vous font gagner un temps précieux. Cet ouvrage complet vous aide dans votre travail quotidien. Il est irremplaçable pour constituer un camet d'adresses, vérifier un téléphone ou un fax, rechercher un confrère ou un établissement. Chaque fiche biographique et professionnelle du médecin ou signalétique de l'établissement est la plus complète possible. Plusieurs classements : alphabétique, géographique, par spécialités et disciplines pernnettent de trouver immédiatement les renseignements précis recherchés.

Depuis des générations, les médecins utilisent le Rosenwald. Ils sont sûrs d'y retrouver rapidement tout ce qu'il faut savoir sur les médecins, les établissements de soins et les professionnels de la Santé.

Guide Rosenwald, 10, rue Vineuse, 75784 Paris Cedex 16

Tél. 44.30.81.00 - Fax 44.30.81.11

Les 3 volumes: I $190 \mathrm{~F}$, le volume $470 \mathrm{~F}$ 